\title{
ALOCAÇÃO DA BIOMASSA E CORRELAÇÕES MORFOFISIOLÓGICAS EM LEGUMINOSAS FORRAGEIRAS COM HÁBITOS DE CRESCIMENTO CONTRASTANTES ${ }^{1}$
}

\author{
Simone Meredith Scheffer-Basso ${ }^{2 *}$; Aino Victor Ávila Jacques ${ }^{3,4}$; Miguel Dall' Agnol ${ }^{3,4}$ \\ ${ }^{2}$ Instituto de Ciências Biológicas - UPF, C.P. 611 - CEP: 99001-970 - Passo Fundo, RS. \\ ${ }^{3}$ Depto. de Plantas Forrageiras e Agrometeorologia - UFRGS, C.P.776 - CEP: 91540-000 - Porto Alegre, RS. \\ ${ }^{4}$ Bolsista CNPq. \\ *Autor correspondente <simone@upf.tche.br>
}

\begin{abstract}
RESUMO: A análise da alocação da biomassa e a correlação entre variáveis morfofisiológicas permitem maior entendimento do estabelecimento, produção e persistência de espécies perenes, em trabalhos de melhoramento genético, manejo e ecologia de plantas forrageiras. Este trabalho teve o objetivo de analisar a alocação de biomassa em leguminosas forrageiras (Adesmia latifolia, A. punctata, A. tristis, Lotus corniculatus, L. uliginosus). O experimento foi conduzido em casa de vegetação durante 210 dias (4000 graus-dia); as plantas foram cultivadas em caixas de $1 \mathrm{~m}^{2}$. Nesse período, foram retiradas amostras periódicas para avaliação dos componentes morfológicos e descrição do hábito das espécies. A alocação da biomassa em raízes, caule e folha foi expressa em percentagem de massa seca. A maior alocação em folhas ocorreu nas espécies estoloníferas, $A$. latifolia $(63,53 \%)$ e $A$. punctata $(61,42 \%)$, que, por sua vez, mostraram a menor alocação em raízes, 10,90 a 14,77 \%, respectivamente. Maior alocação de biomassa em caule $(39,38 \%)$ e raízes $(24,39 \%)$ foi verificada em $L$. uliginosus, espécie rizomatosa. Para todas as espécies, o índice de área foliar (IAF) esteve correlacionado positivamente com número de folhas das hastes secundárias e biomassa aérea. A. latifolia e Lotus spp. são exemplos extremos da relativa importância dos componentes morfológicos na formação do IAF e da biomassa. O padrão de acúmulo e alocação de biomassa nas leguminosas estoloníferas é caracterizado, principalmente, pela produção de folhas, indicando a importância do alongamento e enraizamento dos estolões, enquanto em $A$. tristis e Lotus spp., as frações caule e raízes apresentam igual importância.
\end{abstract}

Palavras-chave: Adesmia, Lotus, índice de área foliar

\section{BIOMASS ALLOCATION AND MORPHOPHYSIOLOGICAL CORRELATIONS IN FORAGE LEGUMES WITH CONTRATING GROWTH HABITS}

\begin{abstract}
The biomass allocation analysis and the correlation among morphophysiological variables allow greater understanding of the establishment, yield and persistence of perennial species in plant breeding projects, management and ecology of forage plants. To analyze the biomass allocation of forage legumes (Adesmia latifolia, A. punctata, A. tristis, Lotus corniculatus, L. uliginosus), an experiment was carried out under greenhouse conditions for 210 days (4000 degree-days); the plants were cultivated in $1 \mathrm{~m}^{2}$ wooden boxes. Plants were harvested to evaluate the morphological components and to describe their growth habit. The root, stem and leaf biomass allocations were expressed as dry mass percentages. The greatest allocation on leaves occurred for stoloniferous species, A. latifolia (63.5\%) and A. punctata $(61.4 \%)$, which presented the smallest allocation for roots, 10.9 and $14.7 \%$, respectively. Largest stem biomass allocation (39.4\%) and roots $(24.4 \%)$ were found for $L$. uliginosus, a species with rhizomes. The leaf area index (LAI) was positively correlated with the number of leaves, secondary stems and aerial biomass. A. latifolia and Lotus spp. are extreme examples of the relative importance of morphological components in the formation of LAl and biomass. The accumulation pattern and forage allocation of stoloniferous legumes are mainly characterized by leaf production, pointing out the importance of stolon elongation and rooting, while for A. tristis and Lotus spp., the stem and root fractions had the same importance.

Key words: Adesmia, Lotus, leaf area index
\end{abstract}

\section{INTRODUÇÃO}

A alocação de recursos em plantas tem sido descrita através da distribuição preferencial de biomassa e nutrientes em seus diversos órgãos. A distribuição desses recursos depende de vários fatores, como idade, nutrição, competição, relações hídricas, hábito de crescimento, etc (Brouwer, 1962). No que se relaciona a plantas cultivadas, visa-se obter diferentes razões de alocação raiz:parte aérea, dependendo de seu hábito de crescimento. Enquanto em plantas produtoras de grãos, um melhor rendimento está associado a uma maior alocação para parte aérea, em espécies forrageiras perenes, é preferível um balanço que permita a sua

${ }^{1}$ Parte da Tese de Doutorado da primeira autora, apresentada à UFRGS, RS. 
persistência. Uma menor relação parte aérea/raízes pode significar um sistema radical mais extenso e, possivelmente, mais eficiente (Buttery \& Bizzell, 1972). Sob pastejo, uma proporção da parte aérea é removida, sendo o primeiro efeito a redução no crescimento radical e a redução no suprimento de carbono fixado, até que o crescimento aéreo seja retomado. Se o pastejo coincidir com períodos em que o solo esteja seco, o desenvolvimento de raízes profundas pode ser limitado (Kemp \& Culvenor, 1994). Nas leguminosas, a presença dos nódulos acrescenta outra dimensão ao problema, porque nessas plantas o sistema de raízes parece ser metabolicamente mais ativo do que em plantas sem nódulos (Ryle et al., 1981). Desta forma, estudos sobre alocação de recursos em plantas forrageiras, devem considerar uma possível influência de nódulos sobre a nutrição e conseqüente distribuição de recursos na planta.

Em espécies nativas, o assunto assume especial importância, uma vez que vegetam normalmente em solos pouco férteis, e o modelo de alocação de biomassa é uma estratégia de sobrevivência. Além disso, a importância de se conhecer o modelo de crescimento de uma espécie permite, portanto, maior entendimento do seu potencial de produção e fornece subsídios aos programas de melhoramento. Assim, este trabalho teve como objetivo analisar a alocação de biomassa em leguminosas forrageiras com diferentes hábitos de crescimento dos gêneros Adesmia DC. (nativo) e Lotus L. (exótico), com a finalidade de contribuir em trabalhos de seleção, manejo e ecologia. Das espécies estudadas, Adesmia latifolia (Spreng.) Vog. e A. punctata (Poir.) DC. são estoloníferas e Lotus uliginosus Schkuhr é rizomatosa, sendo, portanto, espécies clonais. Adesmia tristis Vog. e Lotus corniculatus L. são espécies não clonais, eretas e formadoras de coroa. Todas as espécies se reproduzem por sementes, mas as estoloníferas e a rizomatosa se propagam também através da segmentação dos estolões e rizomas.

\section{MATERIAL E MÉTODOS}

O experimento foi conduzido em casa de vegetação, em Passo Fundo. O município se localiza a $28^{\circ} 15^{\prime} \mathrm{S}, 52^{\circ} 24^{\prime \prime} \mathrm{W}$ e a $687 \mathrm{~m}$ de altitude, na região fisiográfica do Planalto Médio do Rio Grande do Sul. A região possui clima fundamental úmido e variedade subtropical (Cfa). Foram avaliadas cinco espécies (Adesmia latifolia, A. tristis, A. punctata, L. corniculatus cv. São Gabriel e L. uliginosus cv. Grassland Maku), em sete épocas, em delineamento em blocos casualizados, com quatro repetições.

As unidades experimentais consistiram de caixas de madeira forradas com lona plástica perfurada, medindo $0,89 \mathrm{~m} \times 1,00 \mathrm{~m}$ e $0,20 \mathrm{~m}$ de profundidade $\left(0,178 \mathrm{~m}^{3}\right)$, nas quais foram colocados $160 \mathrm{~kg}$ de terra originada de um Latossolo Vermelho-Escuro distrófico, com $610 \mathrm{~g} \mathrm{~kg}^{-1}$ de argila, pH 4,3 e $69 \%$ de saturação por alumínio. O solo pertence à Unidade de Mapeamento Passo Fundo, caracterizado pelo elevado teor de alumínio tóxico e acidez, e com baixa fertilidade natural. O solo corrigido com o equivalente a 12 toneladas de calcário, para elevar o pH a 6,0, e adubado com $390 \mathrm{~kg}$ ha $^{-1}$ de $\mathrm{P}_{2} \mathrm{O}_{5}, 270 \mathrm{~kg}^{-1}$ de $\mathrm{K}_{2} \mathrm{O}$ e micronutrientes (boro, molibdênio, cobre, zinco). Como tratamento fungicida preventivo, foi aplicada uma mistura de quintozene $(75 \%$ PM) a $300 \mathrm{~g}$ de água, metalaxil (35\% PM) a $200 \mathrm{~g} 100$

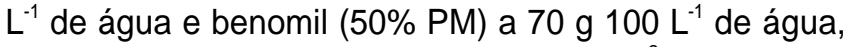
na quantidade de dois litros da calda $\mathrm{m}^{-2}$ de solo; no décimo primeiro dia após a semeadura, foi feita uma segunda aplicação com uma mistura de benomil a 0,05\% e quintozene a $0,3 \%$ (Reis \& Forcelini, 1994). As sementes de Adesmia foram escarificadas em água a $100^{\circ} \mathrm{C}$ durante cinco minutos e colocadas em germinador a $25^{\circ} \mathrm{C}$ durante cinco dias. Em 30 de abril de 1996, as plântulas foram transplantadas para as caixas com solo, sendo dispostas em linhas distantes $0,15 \mathrm{~cm}$, obtendo-se uma população de 100 plantas $\mathrm{m}^{-2}$. Como as plântulas de Lotus não sobreviveram ao transplante, foi realizada a semeadura diretamente no solo em 25 de maio. As sementes de Adesmia e Lotus não foram inoculadas por não se dispor de rizóbio selecionado para as primeiras. O nitrogênio foi suprido na forma de uréia, na dose de 80 $\mathrm{kg} \mathrm{ha}^{-1}$ de $\mathrm{N}$, fracionadas em quatro aplicações. Foi calculado o acúmulo de graus-dia (GD), considerando-se a temperatura basal de $0^{\circ} \mathrm{C}$, segundo Romo \& Eddleman (1995). Tal procedimento teve como objetivo expressar o período de crescimento e desenvolvimento em termos de quantidade de energia térmica acumulada, de maneira a permitir que a seqüência de eventos fosse relacionada, com maior precisão, com as condições ambientais vigentes durante o experimento. Para isso, foram tomadas diariamente, a mesma hora do dia, as temperaturas mínima e máxima no interior da casa de vegetação. A amostragem (colheita das plantas) foi destrutiva, sendo que, nas quatro primeiras, a área da amostra foi de 0,20 $\mathrm{m} \times 0,10 \mathrm{~m}$ e, nas três últimas, de 0,20 $\mathrm{m} \times 0,30 \mathrm{~m}$. As plantas foram separadas em raízes, folhas ativas (verdes), folhas senescentes (amarelas), hastes vegetativas $e$ hastes floríferas. Para avaliação do índice de área foliar (IAF), foram separadas todas as folhas ativas da amostra e realizada a leitura eletrônica da área foliar em medidor marca Licor, modelo 3100A. Procedeu-se à contagem de hastes primárias (eixo caulinar primário) e secundárias (ramificações), folhas ativas e senescentes e gemas, que foram classificadas em gemas basilares e gemas superiores, de acordo com sua posição, abaixo ou acima de $2,5 \mathrm{~cm}$ do colo da planta. Os dados apresentados nesse trabalho foram submetidos à análise de correlação.

\section{RESULTADOS E DISCUSSÃO}

\section{Alocação de biomassa}

O L. corniculatus acumulou a maior biomassa, seguido de A. latifolia e A. tristis (Tabela 1). A. latifolia 
foi a mais precoce e a única que mostrou decréscimo na biomassa total ao final do período de estudo, indicando que atingiu o final de ciclo. A. punctata mostrou o menor acúmulo de biomassa, caracterizando-se por ser espécie de crescimento inicial lento.

As espécies estoloníferas ( $A$. latifolia e $A$. punctata) alocaram maior quantidade proporcional de biomassa para folhas, enquanto as demais se caracterizaram pela maior alocação para caules e raízes (Tabela 2). Em L. uliginosus verificaram-se as maiores alocações para caules $(39,38 \%)$ e raízes (24,39\%). Tal resultado deve-se basicamente ao fato de que seu sistema subterrâneo é vigoroso, formado pela raiz primária e suas ramificações, pelos rizomas e pelas raízes adventícias oriundas desses últimos. A menor alocação de biomassa em raízes nas espécies estoloníferas é um fator que merece especial atenção e, assume relevância para a persistência de leguminosas (Kendall et al.,1994). A. latifolia apresentou a menor relação raiz:parte aérea (RPA), com valores similares ao que Pasumarty et al. (1995) obtiveram em trevo branco (Trifolium repens L.) $(0,16)$, também estolonífero. $A$. latifolia possui um sistema de raízes menos robusto, em relação às demais espécies, composto por raízes finas e superficiais e, na sua grande maioria, adventícias, oriundas dos nós dos estolões, o que indica que sua sobrevivência dependerá de condições de umidade que favoreçam o enraizamento desses caules. Rosengurt

Tabela 1 - Acúmulo de matéria seca de leguminosas forrageiras (Adesmia latifolia, Al; A. punctata. A.p; A. tristis, A.t; Lotus corniculatus, L.c; L. uliginosus, L.u) em sete épocas de crescimento.

\begin{tabular}{lcrrrrr}
\hline $\begin{array}{l}\text { Dia após a } \\
\text { emergência }\end{array}$ & $\begin{array}{c}\text { Graus/ } \\
\text { dia }\end{array}$ & L.c & L.u & A.I & A.p & A.t \\
\hline & \multicolumn{1}{c}{${ }^{\circ} \mathrm{C}$} & ------------- & g MS m$^{-2}$ & ------------- \\
58 & 1037 & 2,75 & 1,40 & 39,25 & 15,13 & 8,50 \\
74 & 1352 & 13,00 & 5,55 & 94,00 & 17,01 & 11,00 \\
90 & 1659 & 36,13 & 18,63 & 169,13 & 25,04 & 35,06 \\
108 & 1946 & 29,92 & 28,96 & 193,75 & 49,09 & 63,65 \\
124 & 2274 & 80,96 & 46,06 & 286,72 & 72,82 & 118,86 \\
152 & 2853 & $203,12193,29$ & 251,56 & 151,88 & 240,15 \\
180 & 3532 & $327,21264,21$ & 204,21 & 171,79 & 262,33 \\
\hline
\end{tabular}

Tabela 2 - Alocação proporcional da biomassa total de Adesmia e Lotus em seus principais componentes e relação raiz:parte aérea (RPA).

\begin{tabular}{|c|c|c|c|c|}
\hline \multirow[t]{2}{*}{ Espécie } & \multicolumn{4}{|c|}{ Alocação proporcional da biomassa } \\
\hline & Folha & Caule & Raiz & RPA \\
\hline & \multicolumn{4}{|c|}{---o-- } \\
\hline Adesmia latifolia & 62,24 & 27,70 & 12,07 & 0,14 \\
\hline A. punctata & 61,42 & 23,91 & 14,77 & 0,20 \\
\hline A. tristis & 42,32 & 36,89 & 20,80 & 0,27 \\
\hline Lotus corniculatus & 43,83 & 32,80 & 23,37 & 0,32 \\
\hline L. uliginosus & 37,86 & 39,38 & 24,39 * & 0,34 \\
\hline
\end{tabular}

(1946) citou um fraco crescimento da espécie quando cultivada em encostas altas, bem como sua sensibilidade às secas estivais. Tal vulnerabilidade também ocorre em trevo branco, em que a falta de um sistema subterrâneo vigoroso é o motivo de baixa sobrevivência em zonas subtropicais, onde normalmente ocorrem verões secos (Mannetje, 1996). Nessa leguminosa, Harris et al. (1983) observaram que os cultivares que entraram no inverno com maior e mais equilibrada alocação de biomassa, quantificada pela relação raiz:parte aérea (RPA), foram capazes de suportar maior peso foliar e sua estrutura genotípica pareceu constituir uma população mais apta a persistir sob condições adversas. Isso indica a importância da seleção de materiais com tal propriedade, em plantas de hábito estolonífero.

Em Lotus sp. mediram-se as maiores RPAs, demonstrando a existência de diferenças interespecíficas citadas por Brouwer (1962), que, todavia, podem ser modificadas por condições externas. Abordagens ecológicas realizadas por Chapin (1980) sugerem que em condições de alta fertilidade, espécies de ambientes férteis têm caracteristicamente baixa RPA, em comparação com espécies que vegetam em áreas de baixa fertilidade. Sob o aspecto de manejo, uma baixa RPA pode implicar em maior dependência da área foliar residual para produzir rebrotes vigorosos e competitivos.

Em L. corniculatus, são comuns as citações a respeito de seu lento crescimento inicial, na maioria das vezes vinculado ao tamanho de suas sementes (Shibles \& MacDonald, 1964). No entanto, como raramente os trabalhos em campo avaliam raízes, é difícil isolar os fatores envolvidos com isso. Genrich et al. (1998), estudando Trifolium ambiguum Bieb, também com crescimento inicial lento, observaram que há uma grande alocação de biomassa em raízes e concluíram que plântulas com essa característica têm alto vigor, mas que a melhoria do estabelecimento dessa leguminosa poderia ser obtida através da seleção de plantas que acumulassem maior quantidade de matéria seca na parte aérea. Todavia, Elias \& Chadwick (1979), ao se referirem ao cornichão, afirmam que é justamente a alta RPA que o torna apto ao cultivo em solos de baixa fertilidade, onde um sistema subterrâneo mais desenvolvido confere maior habilidade de absorção de nutrientes. Nesse sentido, $A$. tristis, com similar modelo de alocação de biomassa (Tabela 2), é considerada uma espécie tolerante à acidez do solo, sendo capaz de utilizar mais eficientemente o $\mathrm{P}$ em relação à A. latifolia (Milan et al., 1991).

\section{Correlações morfofisiológicas}

As diferenças entre as espécies quanto ao processo produtivo decorreram de características morfofisiológicas, que responderam diferentemente às condições ambientais. Através da matriz de correlação gerada com todas as variáveis, foram escolhidas aquelas cujos coeficientes foram considerados mais expressivos para esta discussão (Tabela 3). 
As espécies mais prostradas, A. latifolia, $A$. punctata e L. uliginosus, foram as únicas a mostrar correlação significativa entre a matéria seca da parte aérea e número de folhas. Essa relação é bastante óbvia para a $A$. latifolia, uma vez que a sua biomassa aérea é formada basicamente por estolões e folhas. Portanto, para essas leguminosas, a avaliação do número de folhas, como complemento à determinação do IAF, é um aspecto importante para os estudos de seleção, pois indica que a um aumento no número de folhas corresponde um aumento na produção da matéria seca da parte aérea. Caradus \& McKay (1991) concluíram que o número e o tamanho de folhas foram as variáveis que melhor predisseram a proporção do trevo branco em pastagens. A produção de folhas é um importante atributo para a seleção de trevo branco sob pastejo e essa variável está associada com a ramificação dos estolões (Gibson et al., 1963). Nas demais espécies, não houve correlação para tal variável.

Todas as leguminosas mostraram correlações positivas entre IAF e biomassa da parte aérea, especialmente as clonais $A$. latifolia, $A$. punctata e $L$. uliginosus. Sabendo-se que o crescimento e a produção de uma cultura são determinados pela quantidade de radiação interceptada durante a estação de crescimento, as diferenças de produção de forragem encontradas neste trabalho podem ser explicadas, em parte, pelas diferenças na evolução do IAF. Esse índice tem sido extensivamente explorado nos trabalhos com plantas forrageiras e, juntamente com os níveis de carboidratos não estruturais, tem auxiliado na determinação de práticas de manejo. A correlação entre IAF e produção de biomassa aérea em Adesmia spp. e Lotus spp. é evidente (Figura 1). Em A. latifolia, existe tendência de paralelismo entre essas variáveis, tal como Beinhart (1963) observou em trevo branco. Segundo esse autor, o rápido declínio do IAF reflete, em parte, a expansão das plantas através do alongamento dos estolões. Em
A. latifolia, após os $2574 \mathrm{GD}$, a redução de IAF foi mais acentuada em relação à biomassa da parte aérea. Normalmente, com o aumento do IAF, a produção de folhas diminui mais rapidamente do que a produção de hastes ou produção total de MS (Davidson \& Donald, 1958). Dois tipos de relação podem ocorrer entre o IAF e a produção de matéria seca (Haynes, 1980): (a) numa primeira relação, a produção aumenta à medida que aumenta o IAF até alcançar um valor ótimo, quando então declina e (b) noutro caso, há o estabelecimento de um platô na produção de matéria seca, enquanto o IAF continua a aumentar, como na alfafa (Medicago sativa L.). Neste estudo, observou-se, para A. latifolia e Lotus sp. uma relação paralela entre biomassa e IAF (Figura 1) desde a fase de crescimento ativo até a senescência. Em oposição, um padrão produtivo totalmente diferente foi apresentado por $A$. punctata e $A$. tristis. Nessas espécies, o maior acúmulo de MS ocorreu em períodos mais avançados de desenvolvimento, quando o IAF já estava em decréscimo, ocorrendo alta correlação entre biomassa da parte aérea e de caules (Tabela 3), indicando a importância desse componente na produção de forragem, que, para $A$. tristis é perfeitamente condizente com seu hábito subarbustivo.

Em $A$. tristis e $A$. punctata, a redução do IAF teve menor influência sobre a biomassa acumulada. $\mathrm{Na}$ primeira, observou-se, inclusive, um aumento na quantidade de biomassa acumulada com a redução do IAF. A importância dos caules para a produção de matéria seca de $A$. tristis ficou evidenciada também na correlação positiva entre a número de hastes e a biomassa da parte aérea. Essa correlação foi significativa apenas para $A$. tristis e L. uliginosus (Tabela 3). Para o cornichão, destaca-se a alta correlação da produção de forragem com o número de gemas basilares e a falta de correlação com gemas superiores. No cv. São Gabriel, o desenvolvimento progressivo de hastes da coroa sugere o desenvolvimento ativo de gemas basilares (Araújo \&

Tabela 3 - Coeficientes de correlação parcial (r) para o acúmulo de matéria seca da parte aérea e variáveis morfofisiológicas para Adesmia e Lotus.

\begin{tabular}{lccccc}
\hline \multirow{2}{*}{ Variável } & \multicolumn{5}{c}{ Biomassa da parte aérea } \\
\cline { 2 - 6 } & A. latifolia & A. punctata & A. tristis & L. corniculatus & L. uliginosus \\
\hline F & $0,78^{*}$ & $0,53^{*}$ & $0,38 \mathrm{~ns}$ & $0,09 \mathrm{~ns}$ & $0,34 \mathrm{~ns}$ \\
IAF & $0,86^{*}$ & $0,78^{*}$ & $0,47^{*}$ & $0,55^{*}$ & $0,75^{*}$ \\
BC & $0,57^{*}$ & $0,89^{*}$ & $0,89^{*}$ & $0,77^{*}$ & $0,99^{*}$ \\
BF & $0,93^{*}$ & $0,82^{*}$ & $0,41 \mathrm{~ns}$ & $0,57^{*}$ & $0,75^{*}$ \\
BR & $0,26 \mathrm{~ns}$ & $-0,39 \mathrm{~ns}$ & $0,79^{*}$ & $0,77^{*}$ & $0,96^{*}$ \\
H & $0,19 \mathrm{~ns}$ & $0,57^{*}$ & $0,43 \mathrm{~ns}$ & $0,14 \mathrm{~ns}$ & $0,81^{*}$ \\
Gb & $0,11 \mathrm{~ns}$ & $0,35 \mathrm{~ns}$ & $0,38 \mathrm{~ns}$ & $0,73^{*}$ & $0,58^{*}$ \\
Gs & $0,11 \mathrm{~ns}$ & $0,58^{*}$ & $0,05 \mathrm{~ns}$ & $0,28 \mathrm{~ns}$ & $0,68^{*}$ \\
E & $0,39 \mathrm{~ns}$ & $-0,23 \mathrm{~ns}$ & $0,04 \mathrm{~ns}$ & $0,50^{*}$ & $0,48^{*}$ \\
\hline
\end{tabular}

${ }^{*}(P<0,05) ; \mathrm{ns}(P>0,05)$; BF, BC e BR referem-se ao peso da biomassa seca de caule, folhas e raízes respectivamente; $\mathrm{F}, \mathrm{H}, \mathrm{Gb}$ e $\mathrm{Gs}$ referem-se ao número de folhas ativas, hastes, gemas basilares e gemas superiores, respectivamente; $E$ é a estatura do dossel vegetativo. 

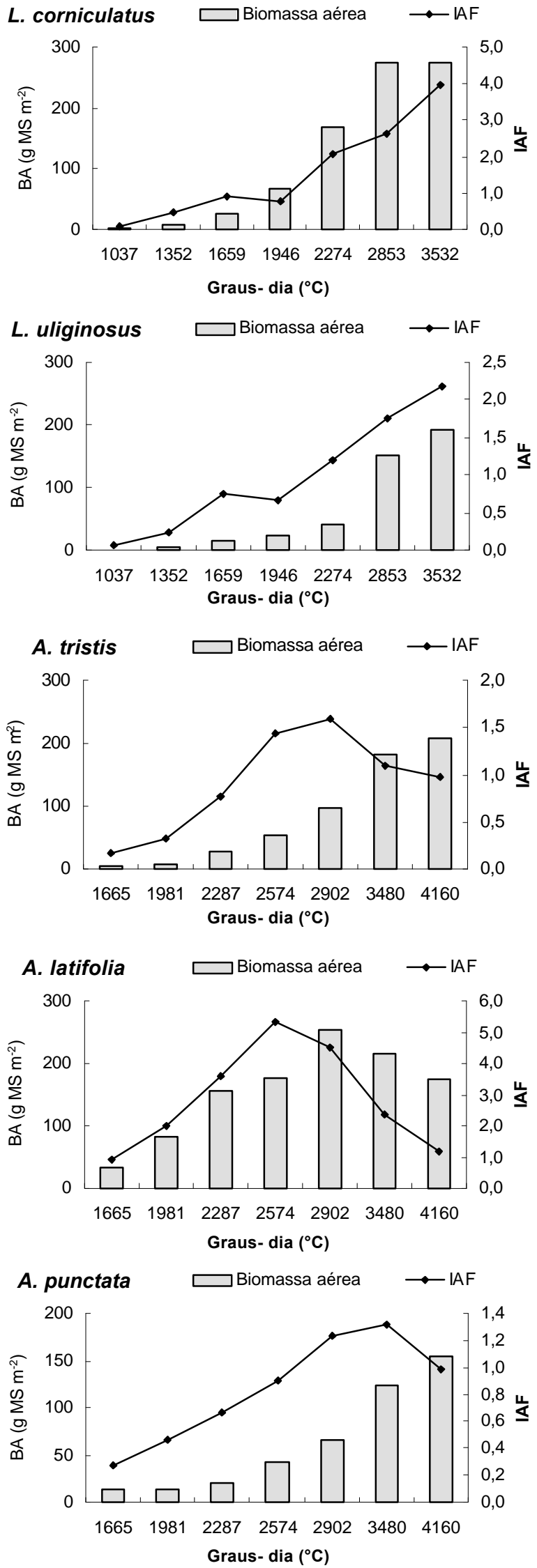

Figura 1 - Dinâmica do acúmulo de biomassa aérea $(B A)$ e do índice de área foliar (IAF) de Adesmia e Lotus ao longo do período de crescimento.
Jacques, 1974), confirmando a validade da correlação encontrada no presente trabalho. Esse fato reforça a importância da avaliação desses pontos de crescimento em trabalhos de melhoramento da espécie, principalmente sob corte ou pastejo, sugerindo que a persistência dessa leguminosa, sob pastejo, deve ser otimizada através da seleção de materiais prostrados, com maiores proporções de hastes oriundas de pontos de crescimento basilares.

O L. uliginosus apresentou uma gama de correlações significativas e de altas magnitudes, e mostrou ser uma espécie cujo processo produtivo depende de vários fatores morfofisiológicos. No entanto, os coeficientes de maior magnitude foram relacionados à produção de caules aéreos, tanto com base na biomassa acumulada, como no número de hastes por $\mathrm{m}^{2}$. Os rizomas só começaram a aparecer aos 153 dias de crescimento, concordando com trabalho de Harris et al. (1997), onde o crescimento inicial da espécie se fez mediante caules aéreos, o que ocorre normalmente na primavera e início de verão. Destaca-se que $L$. uliginosus foi a única espécie a não florescer, o que reforça as conclusões de Paim \& Riboldi (1991) sobre os problemas de adaptação do cv. Maku no Brasil, o qual só costuma florescer no segundo ano.

\section{CONCLUSÕES}

Leguminosas forrageiras com hábitos de crescimento contrastantes mostram características distintas de alocação de biomassa em folhas, caules e raízes, que podem auxiliar a explicar sua distribuição ecológica, produção e persistência em condições de campo. O padrão de acúmulo e alocação de biomassa nas espécies estoloníferas é caracterizado, principalmente, pela produção de folhas, indicando a importância do alongamento e enraizamento dos estolões, enquanto em A. tristis e Lotus spp., as frações caule e raízes apresentam igual importância.

A persistência de uma pastagem de A. latifolia depende da contínua formação de estolões, enquanto em A. tristis e Lotus corniculatus, a sobrevivência do indivíduo é fundamental.

\section{REFERÊNCIAS BIBLIOGRÁFICAS}

ARAÚJO, J.C.; JACQUES, A.V.A. Características morfológicas e produção de matéria seca de cornichão (Lotus corniculatus L.) colhido em diferentes estádios de crescimento e a duas alturas de corte. Revista da Sociedade Brasileira de Zootecnia, v.3, p.138-147, 1974.

BEINHART, G. Effects of environment on meristematic development, leaf area and growth of white clover. Crop Science, v.3, p.209-219, 1963.

BROUWER, R. Distribution of dry matter in the plant. Netherlands Journal of Agricultural Science, v.10, p.361-376, 1962.

BUTTERY, B.R.; BIZZELL, R.I. Some differences between soybean cultivars observed by growth analysis. Canadian Journal of Plant Science, v.52, p.13-20, 1972.

CARADUS, J.R.; McKAY, A.C. Performance of white clover cultivars and breeding lines in a mixed species swards. 2. Plant characters contributing to differences in clover production. New Zealand Journal of Agricultural Research, v.34, p.155-160, 1991.

CHAPIN, F.S. The mineral nutrition of wild plants. Annual Review of Ecology and Systematics, v.11, p.233-260, 1980. 
DAVIDSON, J.L.; DONALD, C.M. The growth of swards of subterranean clover with particular reference to leaf area. Australian Journal of Agricultural Research, v.9, p.53-72, 1958.

ELIAS, C.O.; CHADWICK, M.J. Growth characteristics of grass and legume cultivars and their potential for land reclamation. Journal of Applied Ecology, v.16, p.537-544, 1979.

GENRICH, K.C.; SHEAFFER, C.C.; EHLKE, N.J. Kura clover growth and development during the seeding year. Crop Science, v.38, p.735-741, 1998.

GIBSON, P.B.; BEINHART, G.; HALPIN, J.E. et al. Selection and evaluation of white clover. I. Basis for selection and a comparison of two methods of propagation for advanced evaluations. Crop Science, v.3, p.83-86, 1963.

HARRIS, W.; RHODES, I.; NEE, S.S. Observations on environmental and genotypic influences on the over wintering of white clover. Journal of Applied Ecology, v.20, p.609-624, 1983

HAYNES, R.J. Competitive aspects of the grass-legume association. Advances in Agronomy, v.33, p.227-261, 1980.

KEMP, D.R.; CULVENOR, R.A. Improving the grazing and drought tolerance of temperate perennial grasses. New Zealand Journal of Agricultural Research, v.37, p.365-378, 1994

KENDALL, W.A.; SHAFFER, J.A.; HILL Jr., R.R. Effect of temperature and water variables on the juvenile growth of lucerne and red clover. Grass and Forage Science, v.49, p.264-269, 1994.

MANNETJE, L. Persistence de leguminosas forrajeras en pastures. In: REUNIÓN LATINOAMERICABA DE RHIZOBIOLOGIA, 18., Santa Cruz de la Sierra, 1996. Memorias. Santa Cruz de la Sierra, 1996. p.31-41.
MILAN, P.A.; RITTER, W.; DALL' AGNOL, M. Seleção de leguminosas forrageiras tolerantes a alumínio e eficientes na utilização de fósforo. I. Leguminosas nativas. Pesquisa Agropecuária Brasileira, v.26, p.119124, 1991.

PAIM, N.R.; RIBOLDI, J. Competição entre espécies e cultivares do gênero Lotus L. Pesquisa Agropecuária Brasileira, v.26, p.1699-1704, 1991.

PASUMARTY, S.V.; HIGUCHII, S.; MURATA, T. et al. Influence of seed quality on seedling growth of white clover (Trifolium repens L.) Grass and Forage Science, v.50, p.93-97, 1995

REIS, E.M.; FORCELINI, C.A. Manual de fungicidas: guia para o controle de doenças de plantas. Passo Fundo: Pe. Berthier, 1994. 100p.

ROMO, J.T.; EDDLEMAN, L.E. Use of degree-days in multiple-temperature experiments. Journal of Range Management, v.48, p.410-416, 1995.

ROSEGURT, B. Gramineas y leguminosas de Juan Jackson - Comportamiento en el campo y en cultivo. In: ROSENGURT, B. (Ed.) Estudos sobre praderas naturales del Uruguay - $5^{\mathrm{a}}$ Contribución. Montevideo: Imprenta Rosgal, 1946. p.215-346.

RYLE, G.J.A.; POWELL, C.E.; GORDON, J. Assimilate partitioning in red and white clover either dependent on $\mathrm{N}_{2}$ fixation in root nodules or utilizing nitrate nitrogen. Annals of Botany, v.47, p.515-523, 1981.

SHIBLES, R.M.; MacDONALD, H.A. Photosynthetic area and rate in relation to seedling vigor of birdsfoot trefoil (Lotus corniculatus L.) Crop Science, v.2, p.299-302, 1964.

Recebido em 21.11.01 